\title{
Experimental Results of Near Real-Time Protection System for Plasma Facing Components in Wendelstein 7-X at GLADIS
}

\author{
A. Ali, ${ }^{1,2, \text { ศ } M . ~ J a k u b o w s k i, ~}{ }^{1}$ H. Greuner, ${ }^{3}$ B. Böswirth,${ }^{3}$ V. Moncada,${ }^{4}$ \\ A. Puig Sitjes, ${ }^{1}$ R. Neu, ${ }^{2,3}$ T. S. Pedersen, ${ }^{1}$ and the W7-X Team ${ }^{1}$ \\ ${ }^{1}$ Max Planck Institute for Plasma Physics, Wendelsteinstraße 1, 17491 Greifswald, Germany \\ ${ }^{2}$ TUM, Department of Mechanical Engineering, Boltzmannstraße 15, 85748 Garching, Germany \\ ${ }^{3}$ Max Planck Institute for Plasma Physics, Boltzmannstraße 2, 85748 Garching, Germany \\ ${ }^{4}$ Thermadiag, 100 Impasse des Houilléres, ZA Le Pontet, F-13590 Mayreuil, France
}

\begin{abstract}
One of the aims of stellarator Wendelstein 7-X (W7-X), is to investigate steady state operation, for which power exhaust is an important issue. The predominant fraction of the energy lost from the confined plasma region will be absorbed by an island divertors, which is designed for $10 \mathrm{MWm}^{-2}$ steady state operation. In order to protect the divertor targets from overheating, 10 state-of-the-art infrared endoscopes will be installed at W7-X.

In this work, we present the experimental results obtained at the high heat flux test facility GLADIS (Garching LArge DIvertor Sample test facility in IPP Garching) [1] during tests of a new plasma facing components (PFCs) protection algorithm designed for W7-X. The GLADIS device is equipped with two ion beams which can generate a heat load in the range from $3 \mathrm{MWm}^{-2}$ to $55 \mathrm{MWm}^{-2}$. The algorithms developed at W7-X to detect defects and hot spots are based on the analysis of surface temperature evolution and are adapted to work in near real-time. The aim of this work was to test the near real-time algorithms in conditions close to those expected in W7-X. The experiments were performed on $\mathrm{W} 7-\mathrm{X}$ pre-series tiles to detect $\mathrm{CFC} / \mathrm{Cu}$ delaminations. For detection of surface layers, Carbon Fiber Composite (CFC) blocks from the divertor of Wendelstein 7-AS stellarator were used to observe temporal behavior of fully developed surface layers.

These layers of re-deposited materials like carbon, boron, oxygen and iron were formed during the W7-AS operation. A detailed analysis of the composition and their thermal response to high heat fluxes (HHF) are described in [2] . The experiments indicate that the automatic detection of critical events works according to W7-X PFC protection requirements.
\end{abstract}

\footnotetext{
* author's email adnan.ali@ipp.mpg.de
} 


\section{INTRODUCTION}

W7-X is an optimized stellarator, designed for steady state operation of up to 30 minutes. The PFCs which are subject to largest power loads include divertors and first wall. During operation, these components have to be actively water cooled. A possible overheating of PFCs may lead to defects at the bonding of the CFC tiles to the Active Metal Casting (AMC) interlayer [3]. Thus, it is required to detect such defects in near real-time during plasma operation in order to reduce the local heat flux if required. Such systems are being also developed in other fusion devices, e.g. JET [4] and ASDEX Upgrade [5] 6]. This paper provides a detailed explanation of the RTPS (Real Time Protection System) framework and its test at GLADIS. Section II provides a description of the CFC which is used in the divertor target element. Section III describes the RTPS framework and its integration with the main central control system. Section IV explains the experimetal setup at GLADIS. Section V explains the results obtained from the experiments conducted in GLADIS. Section VI gives a short outlook and a summary.

\section{CARBON FIBER COMPOSITE (CFC)}

The divertor of W7-X is subdivided in units which are composed of about 890 PFCs covered with about 14000 flat tiles of CFC NB31[7]. These CFC tiles are bonded via a AMC-Cu interlayer to a copper chromium zirconium $(\mathrm{CuCrZr})$ cooling structure. The basic design of the target elements can be seen in Figure 1.

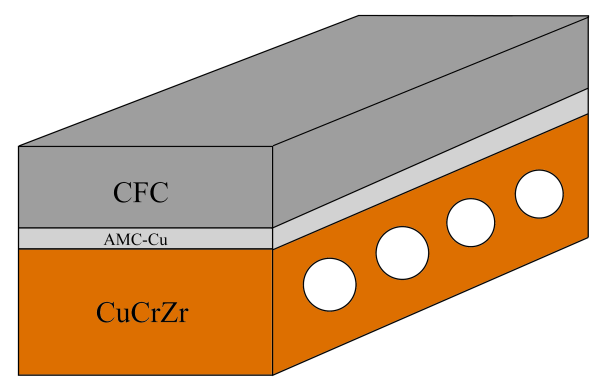

FIG. 1: Schematic design of W7-X divertor target element. The CFC tile is bonded with the $\mathrm{CuCrZr}$ cooling structure via AMC-Cu interlayer.

These target elements are designed to withstand a steady state heat flux of upto $10 \mathrm{MWm}^{-2}$ for long term plasma operations of W7-X [7]. Each divertor module consists of up to $250 \mathrm{~mm}$ 
to $500 \mathrm{~mm}$ long and $50 \mathrm{~mm}$ to $61.5 \mathrm{~mm}$ wide individual target elements which are covered with an on average $6 \mathrm{~mm}$ thick $\mathrm{CFC}$ top tile.

In the past, many experiments were conducted in GLADIS to assess the performance of prototypes of W7-X target elements during high heat loads. In general three different types of CFC tile bonding defects (so-called corner, enclosed and band defect depending on the local appearance) were detected during IR analysis and later confirmed by metallographic examination of the prototype target elements. All defects occurred at the interface between the $\mathrm{CFC}$ and $\mathrm{AMC}$ interlayer and are named delaminations. Further details regarding the formation of these defects are discussed in [8].

In order to detect above mentioned defects, a method [3] was developed based on the surface temperature decay time defined as

$$
\tau=\frac{T(t)}{\partial_{t} T(t)}
$$

A so-called $\tau$ criterion $\left(\tau>\tau_{\text {crit }}\right.$ where $\left.\tau_{\text {crit }}=\tau_{\text {mean }}+4 \sigma\right)$ was evaluated by observing behavior of the target elements under different heat loads with an infrared diagnostic. The method defined in [3] was developed to detect thermal events using the GPU. However, the focus of the efforts lies on significantly improving the efficiency of the parallel computing, which allows to process 100 times more data at the same time.

The probability of sending false positive alarms would be higher if the criterion for sending alarms would be based on the evaluation of only a single pixel within the defect area. Although the defects are being detected in the system using the method described in [3], determining the size of the defects on the material is also an important requirement to reduce the amount of false positive alarms.

For this reason, an additional algortihm, which is based on the concept of component connected labeling (CCL) has been developed. A similar method has also been applied in [9], where also a labelling is made based on the binary results obtained from the GPU. CCL is a fast search algorithm where a 4 or 8 point search (up, down, left, right and diagonal elements) is applied. Initially all elements of the label array are assigned index labels with respect to their location. For each group of pixels where defects were detected, all the pixels within the group are labeled by the smallest pixel index chosen from all the pixels within this group. In this way all the pixels which are connected to each other are assigned to a single label value which can be seen be in Figure 2. The next step is to calculate the size of 
the defect, which can be used as an additional criterion for generating trigger signals, i.e. send a warning signal if the defect size is larger than $n$ number of pixels.

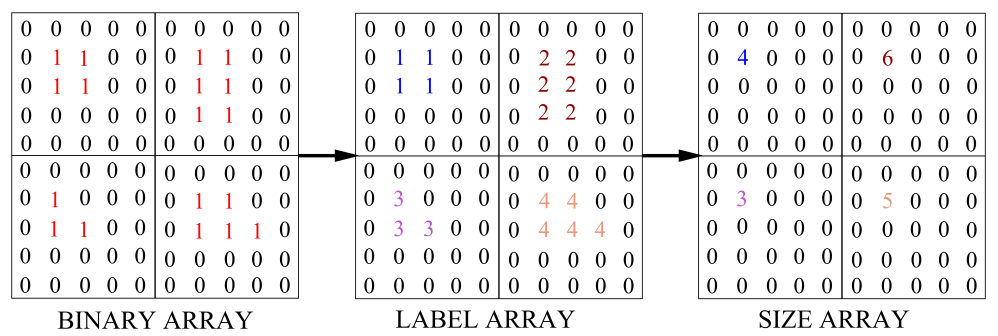

FIG. 2: The resultant binary array for surface layers, delaminations and hotspots is mapped to label array and then size of each label is calculated in GPU.

\section{RTPS FRAME WORK}

The primary goal of $\mathrm{W} 7-\mathrm{X}$ is to maintain a steady-state plasma for 30 minutes. On such time scales, defects can be also formed within a single discharge as mentioned in [3], [8]. For this reason, the RTPS is designed to create an early response system to provide feedback information to the W7-X central safety system (CSS) if an overheating or defect are detected on any of the observed PFCs. The block diagram of the system is presented in Figure 3 . The data is processed in parallel and assigned to different software modules i.e. visualiziation of the surface temperatures, processing the data with GPU to look for defects and saving the data to a hard disk.

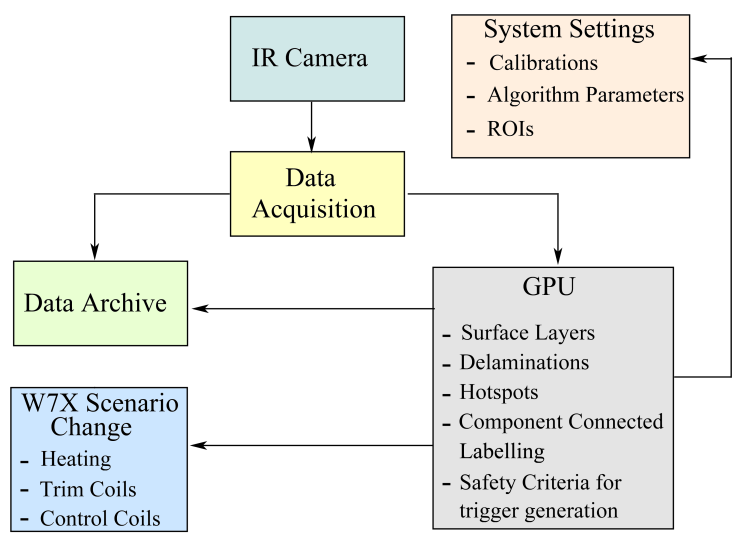

FIG. 3: Block diagram of the RTPS designed for the protection of PFCs in W7-X by detecting thermal events in the infrared images using GPU algorithms. 
During the discharge, the system classifies the data to detect thermal events. The IR camera sends the raw data to the data acquisition system which then generates the parallelized tasks for the CPU and GPU. For parallel processing, an NVIDIA Quadro graphics processing unit (GPU) is used. Advanced GPU computation techniques were used to achieve higher performance. A data acquisition card (DAQ) is used to send/receive trigger signals. A camlink interface card is used to acquire data from an IR camera.

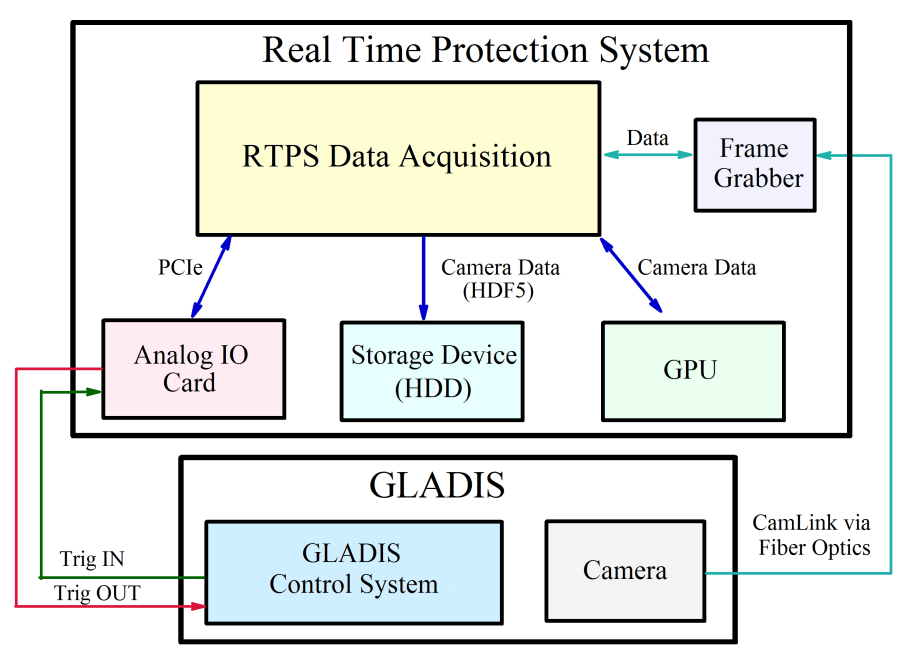

FIG. 4: Hardware setup of the RTPS at GLADIS. The camera sends the data to the RTPS data acquisition system where it performs parallel task of recording and analyzing the IR data. If critical events are detected by the RTPS, a trigger OUT signal was generated by

RTPS which was received by GLADIS data acquisition system

Additional attention has been given to so-called surface layers [2], which are formed by poorly attached thin (a few microns) layers of hydrocarbons on the surface of PFCs due to erosion and redeposition of the carbon. Although they are not posing any danger to the machine, they lead to false surface temperatures measured by thermography and thus to overestimated values of the heat flux density. This has been observed in virtually every device with carbon PFCs e.g. W7-AS [2], JET [4] and Tore Supra [10]. Overestimated surface temperature would lead to false positive alarms. Therefore a prerequisite for smooth steady state operation in fusion devices with water-cooled carbon PFCs is to detect surface layers and apply corrections on a regular basis. This has also been tested during this work. 


\section{EXPERIMENTAL SETUP AT GLADIS}

In order to qualify if the RTPS is compatible with the safety requirements of W7-X, HHF experiments on W7-X prototype divertor target elements were conducted at GLADIS [1]. GLADIS is a facility to test plasma facing components under high heat loads with heat flux densities up to $55 \mathrm{MWm}^{-2}$. To investigate if the RTPS is able to detect hotspots, surface layers and delaminations in near real-time, a pre-damaged prototype target element of an early pre-series (4S-032) was tested under different heating scenarios. Additionally, CFC blocks of Wendelstein 7-AS similar to those discussed in [2] and [11] were used to detect surface layers of re-deposited materials, e.g. carbon, boron, oxygen and iron [2]. The main part of the RTPS is an IR camera measuring in the wavelength range of $3 \mu \mathrm{m}$ to $5 \mu \mathrm{m}$ with an InSb intrinsic semiconductor sensor. During experiments at GLADIS, the spatial resolution of IR camera was $4 \mathrm{~mm} /$ pixel, which is of the same order as expected for thermographic systems built for W7-X. An additional sapphire filter was used to prevent saturation of the infrared sensor. In order to simulate a realistic scenario for W7-X, the heating power was varied between $3,5,8$ and $10 \mathrm{MWm}^{-2}$.

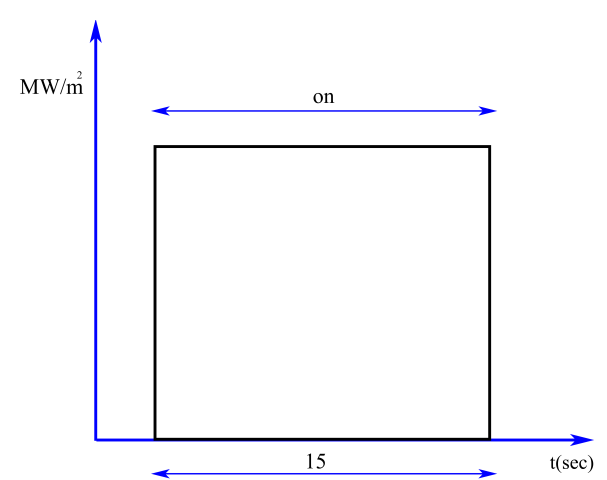

(a) Constant beam discharge

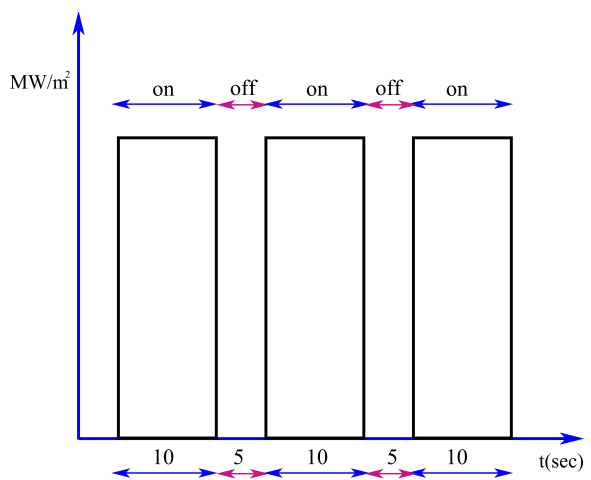

(b) Modulated discharge

FIG. 5: Two different types of operation i.e. constant and modulated beam loading were used. The heating power and duration of the beam loads were varied to have different temperature profiles on the target element

Two different types of operation (constant and modulated heating power) were used as seen in figure 5. The beam focus on the W7-X target element was also varied between tile 4, 6 and 8 as can be seen in Figure 6 to detect the defects with direct and indirect beam focus on the defected tiles . 


\section{EXPERIMENTAL RESULTS}

As shown in [3] the detection of delaminations is difficult if the heat load is less than $8 \mathrm{MWm}^{-2}$. In this work we have used power loads of up to $10 \mathrm{MWm}^{-2}$, which allowed for clear detection of delaminated tiles. An example representing surface temperature on CFC tiles during and after the heating cycle is presented in Figure 6 .

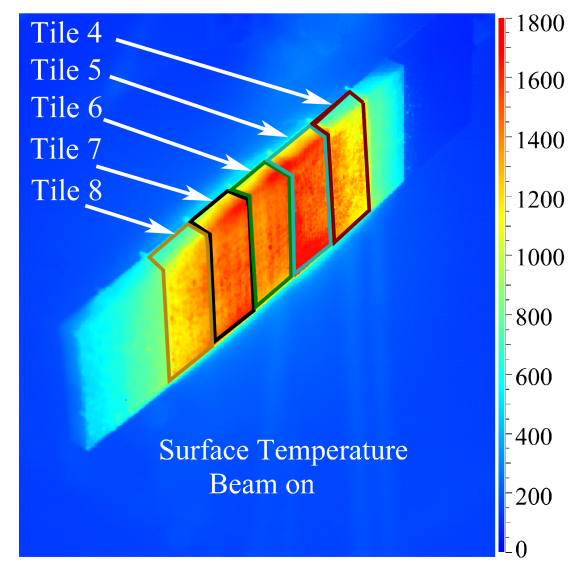

(a) Surface Temperature Beam on

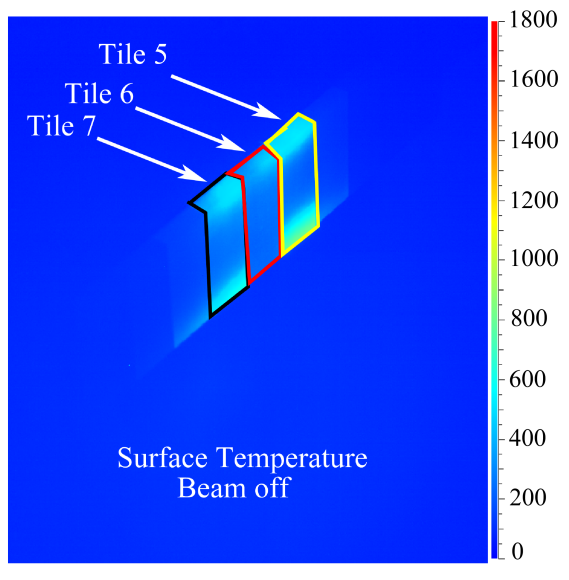

(b) Surface Temperature Beam off

FIG. 6: False color image of surface temperature on one of the W7-X divertor target elements tested in GLADIS. Figure 6a show surface temperature on the CFC tiles during

the beam injection with heat loads of up to $10 \mathrm{MW} / \mathrm{m}^{2}$. Figure $6 \mathrm{~b}$ show the surface temperature after beam is turned off. Regions of interest (ROIs) in Figure 6b in yellow, red and black represent the areas of tile 5, 6 and 7 .

The surface temperature on tiles 5 and 7 in Figure 6 appears to be higher as compared to the tiles without any defects (i.e. 4,8). Measured surface temperature is passed through an image processing algorithm to create a binary output arrays with the information on detected defects. An example processed for data presented in Figure 6 can be seen in Figure 7, where detected delaminations are marked in red. The system creates 5 independent output matrices with information on detected surface layers, delaminations and hotspots. The 4th output matrix shows the output of CCL algorithm. The 5th one reports the size of the corresponding defects (as presented in Figure 7). These values are compared to a threshold value defined by the user. If the calculated size of a defect exceeds the threshold, a trigger signal is generated by RTPS and then it is sent to GLADIS control system where it is recorded. 


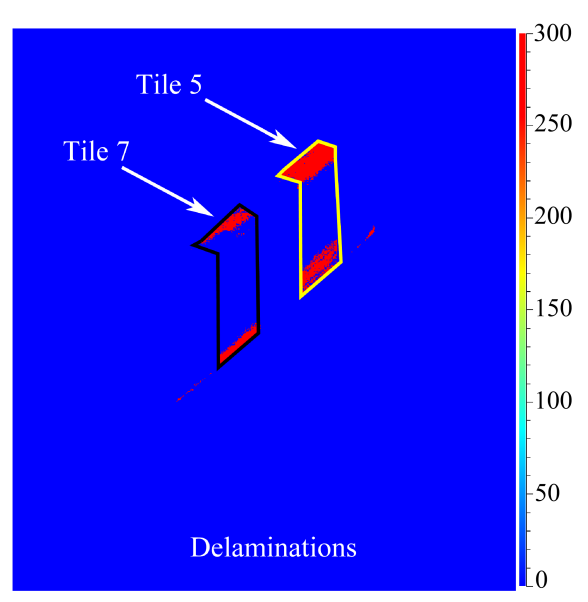

(a) Delaminations

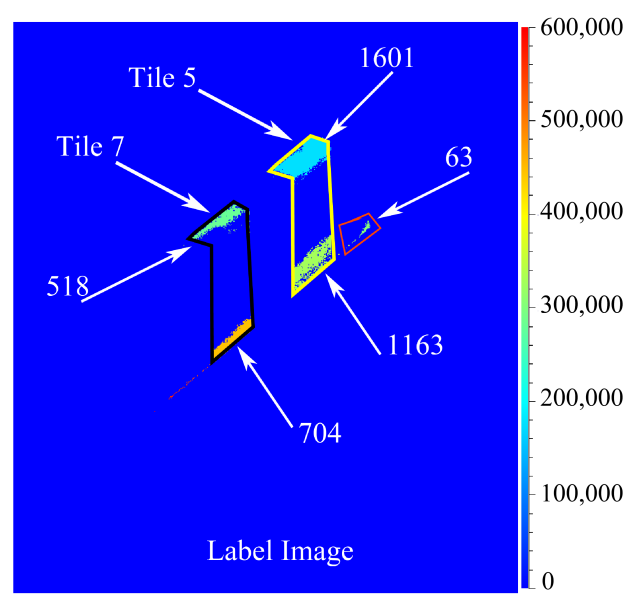

(b) Label image

FIG. 7: Binary output arrays obtained from image processing algorithm in GPU. Figure 7a show the delaminations detected at the end of the operation. Figure $7 \mathrm{~b}$ show Label image where ROIs in yellow and black represent the area of tile 5 and 7 . The numbers on Label image represents the size of each label calculated by 5th data array.

As mentioned in IV, W7-AS divertor tiles were used to provide realistic conditions for the detection of surface layers. After the visual inspection of the W7-AS divertor modules, tile 5, 12 and 13 with maximum deposition were selected (see Figure 8). For further details on deposits on W7-AS PFCs see [2] and [11].

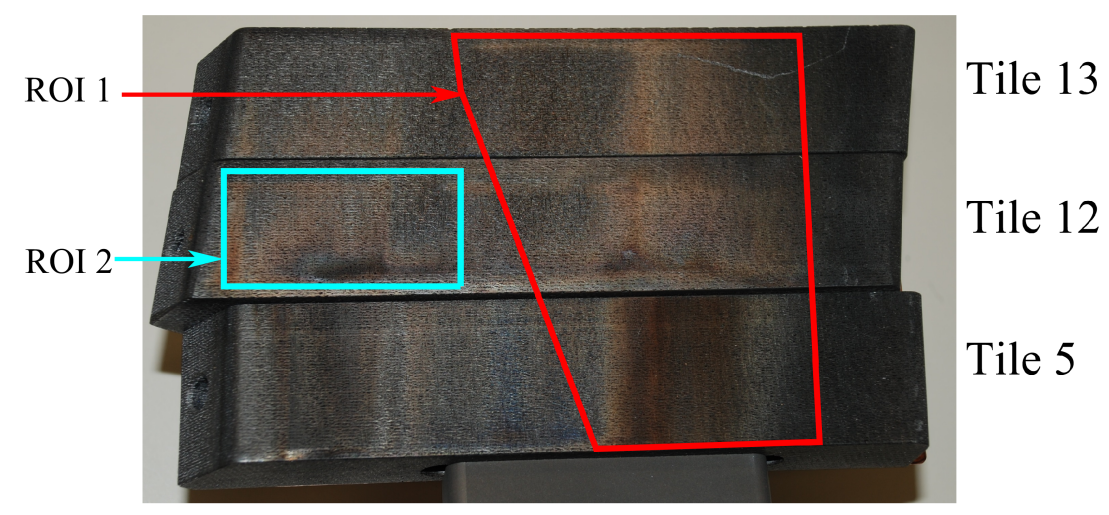

FIG. 8: W7-AS divertor target elements. The numbers indicate the tiles position on the entire divertor module. The ROIs show the areas where the surface layers are present.

In order to test detection of the surface layers by RTPS a modulated heat loads with square waveform and amplitude of $10 \mathrm{MWm}^{-2}$ were applied. The output of the algorithm is 
presented in Figure 9. The surface layers have been detected on all the tiles and they are marked in red color in Figure 9b. This information will be used in future as an input for algortithms looking for overheated areas.

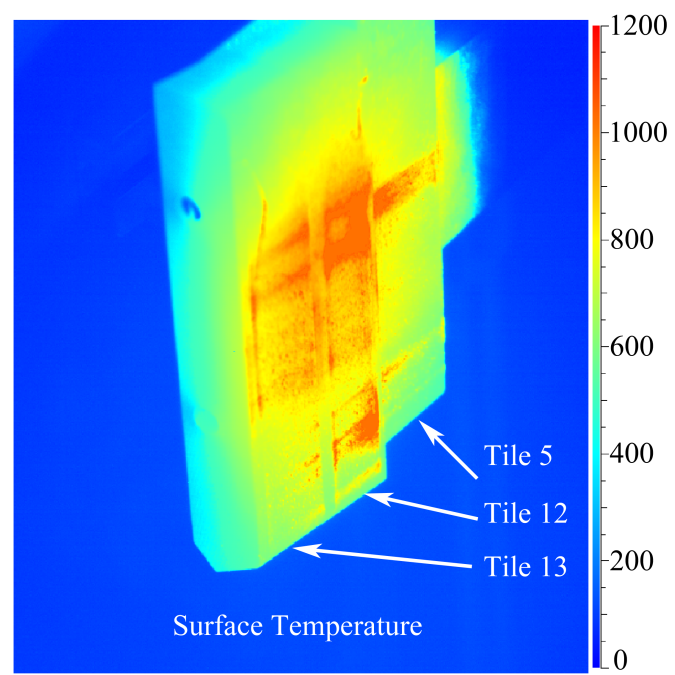

(a) Surface Temperature

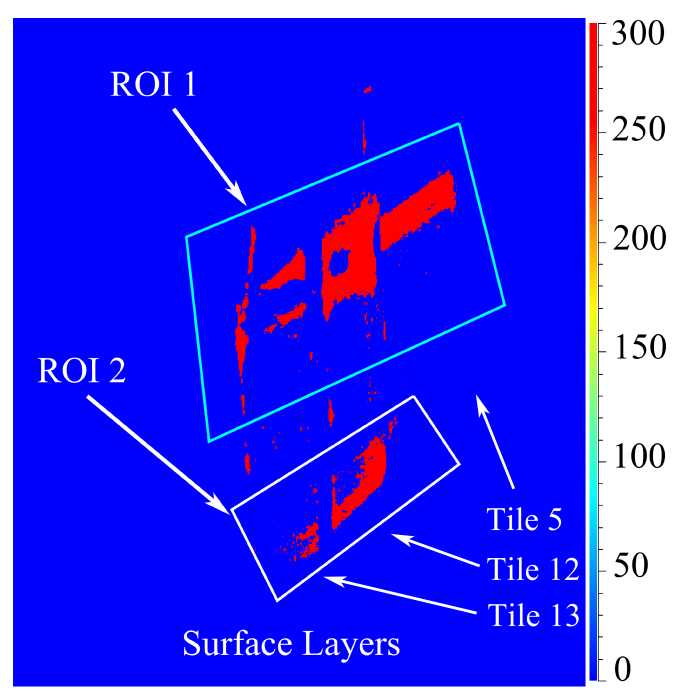

(b) Surface Layers

FIG. 9: False color image of W7-AS divertor target element. Figure 9a show surface temperature on the CFC tiles during the heat loading. Figure $9 \mathrm{~b}$ show the surface layers detected by the system. ROIs in blue and white in Figure 9b represents the areas with expected deposition of surface layers.

\section{OUTLOOK AND SUMMARY}

In this work, we presented the experimental performance of the RTPS at GLADIS for detection of surface layers, delaminations and hotspots of different origins. Algorithms are developed to detect these defects in near real-time based on thermographic data. The results presented in the paper clearly show that the system was able to detect these defects in near real-time and it is ready for implementation and tests at W7-X, which is now undergoing. Surface layers and hotspots are expected to be detected in the next experimental campaign when inertially cooled divertors are used. However, delaminations will be detected when water-cooled high-heat-flux divertors are installed during experimental campaign in 2020. 


\section{ACKNOWLEDGEMENT}

This work has been carried out within the framework of the EUROfusion Consortium and has received funding from the Euratom research and training programme 2014-2018 under grant agreement No 633053. The views and opinions expressed herein do not necessarily reflect those of the European Commission.

\section{REFERENCES}

[1] H. Greuner, B. Boeswirth, J. Boscary, and P. McNeely, Journal of Nuclear Materials 367, 1444 (2007).

[2] D. Hildebrandt, A. Dübner, H. Greuner, and A. Wiltner, Journal of Nuclear Materials 390, 1118 (2009).

[3] A. Rodatos, H. Greuner, M. Jakubowski, J. Boscary, G. Wurden, T. Pedersen, and R. König, Review of Scientific Instruments 87, 023506 (2016).

[4] A. Huber and others (to be published), Physica Scripta 2017 (2017).

[5] A. Herrmann, R. Drube, T. Lunt, and P. de Marné, Fusion Engineering and Design 86, 530 (2011), proceedings of the 26th Symposium of Fusion Technology (SOFT-26).

[6] B. Sieglin, M. Faitsch, A. Herrmann, B. Brucker, T. Eich, L. Kammerloher, and S. Martinov, Review of Scientific Instruments 86, 113502 (2015).

[7] J. Boscary, A. Peacock, T. Friedrich, H. Greuner, B. Böeswirth, H. Tittes, W. Schulmeyer, and F. Hurd, Fusion Engineering and Design 87, 1453 (2012), tenth International Symposium on Fusion Nuclear Technology (ISFNT-10).

[8] H. Greuner, B. Böswirth, J. Boscary, P. Chaudhuri, J. Schlosser, T. Friedrich, A. Plankensteiner, and R. Tivey, Journal of Nuclear Materials 386, 772 (2009).

[9] O. Kalentev, A. Rai, S. Kemnitz, and R. Schneider, Journal of Parallel and Distributed Computing 71, 615 (2011).

[10] M. Mitteau, J. C. Vallet, R. Reichle, C. Brosset, P. Chappius, J. J. Cordier, E. Delchambre, F. Escourbiac, A. Grosman, D. Guilhem, M. Lipa, T. Loarer, J. Schlosser, and E. Tsitrone, Physica Scripta 2004, 157 (2004). 
[11] D. Hildebrandt, F. Gadelmeier, P. Grigull, K. McCormick, D. Naujoks, D. Sünder, et al., Journal of nuclear materials 313, 738 (2003). 